\title{
Semiclassical Effects and the Onset of Inflation
}

\author{
Esteban Calzetta \\ 世 Instituto de Astronomía y Física del Espacio \\ cc 67, suc 28, (1428) Buenos Aires, Argentina \\ Maria Sakellariadou \\ 田用 Research Group on General Relativity \\ Faculté des Sciences, Université Libre de Bruxelles \\ CP 231 Campus Plaine, Boulevard du Triomphe, 1050 Bruxelles, Belgium \\ (Received )
}

\begin{abstract}
We present a class of exact solutions to the constraint equations of General Relativity coupled to a Klein - Gordon field, these solutions being isotropic but not homogeneous. We analyze the subsequent evolution of the consistent Cauchy data represented by those solutions, showing that only certain special initial conditions eventually lead to successfull Inflationary cosmologies. We argue, however, that these initial conditions are precisely the likely outcomes of quantum events occurred before the inflationary era.
\end{abstract}

98.80.Cq,98.80.Dr

Typeset Using REVTEX 


\section{INTRODUCTION}

In this paper we address the issue of the relevance of semiclassical cosmological effects to Inflation in the Very Early Universe. To this end, we consider spherically symmetric models in which the geometry is coupled to a massive, free, minimally coupled real scalar field. We propose a particular choice of gauge, which allows us to solve Einstein's constraint equations ( see below ) exactly, in closed form, in the case of interest. We then use the self consistent Cauchy data so obtained to discuss the necessary conditions for "natural" Inflation (see below), and match these conditions against those likely to result out of a semiclassical era. From this analysis, we conclude that semiclassical effects enhance the likelihood of Inflation.

The combination of the Hot Big Bang Model and Inflation provides at present the most comprehensive picture of the evolution of the Universe at our disposal. To the well known successfull quantitative predictions of the Big Bang model ( such as the existence and temperature of the Cosmic Microwave Background, the relative abundances of light elements and the red shift of distant galaxies [1] ), Inflation adds a plausible explanation for the near critical value of the observed density in the Universe [2]. It also predicts a primordial fluctuations spectrum whose amplitude has the right order of magnitude, as checked against recent observations [3] However, this class of cosmological models rests on the assumption of highly specific initial conditions for the cosmic evolution. This situation is hardly acceptable, specially because alleviating the fine tuning of initial conditions, as required by the Big Bang model, was historically one of the motivations to investigate inflationary cosmologies 4.

It should be stressed that the proper study of the necessary conditions for Inflation requires the consideration of nonlinear effects; indeed, linear perturbations of an Inflationary solution are eventually redshifted away, and cannot affect the global characther of the cosmic evolution [5]. However, nonlinear perturbations can decouple themselves from the Hubble flow and destroy the homogeneity that Inflation is supposed to bring about.

Non linear perturbations of an Inflationary model have been considered by a number 
of authors [6], most notably in a series of numerical simulations by Goldwirth and Piran [7]. These analysis concur in that Inflation demands initial conditions which are already homogeneous over regions larger than the original horizon size. This homogeneity cannot be accounted for through ( classical ) physical processes acting in the pre - Inflationary era. Therefore, to achieve successfull Inflation in classical cosmology, some fine tuning of initial conditions cannot be avoided.

The details of how initial conditions should be fixed vary with the different versions of the inflationary model in the literature. In this paper, we shall consider only the "chaotic" inflationary model [8], where Inflation is sustained by the stress energy of a free Klein Gordon field, in an Universe born from an initial singularity. We choose these models because their extreme simplicity makes them the most universal of inflationary cosmologies.

The limitations on initial conditions required by chaotic inflation can be understood in a back of the envelope calculation, as follows. Suppose an inflaton field $\phi$ of mass $m$, varying over distances $\lambda$, being the source for an inflationary expansion with Hubble constant $H \sim m \phi / m_{p}$, where $m_{p}$ stands for Planck's mass . The condition of vacuum dominance requires $m \lambda \gg 1$ and $\dot{\phi} \ll m \phi$, where a dot is a derivative with respect to cosmological time. On the other hand, under conditions of slow roll over, $\dot{\phi} \sim m^{2} \phi / H$, from where we get $\phi \gg m_{p}$, and $\lambda \gg m^{-1} \gg H^{-1}$. A more carefull analysis shows that $\lambda$ should be some ten times the horizon size [9].

While this result shows that Inflation in no way frees the present state of the Universe from dependence on initial conditions, the question remains about the relative likelihood of inflationary initial conditions as opposed to generic ones. Nevertheless, here too, as long as we remain within the framework of classical cosmology, Inflation proves itself of little use. General Relativity being a time reversal invariant theory, final and initial configurations are in a one to one correspondence. Therefore no mechanism such as Inflation can enhance the likelihood of a particular set of final states ( of course, this holds as long as one does not introduce an ad hoc measure in configuration space ). In other words, the inflationary hypothesis does not render a homogeneous and isotropic Universe any more likely than 
simply to assume homogeneity and isotropy to begin with.

The situation changes when semiclassical effects are taken into account. Indeed, while quantum fields in curved spaces evolve unitarily, it is generally impossible in concrete situations to determine exactly the quantum state of a given field. For example, while we know the occupation numbers of the different modes in the Background Microwave Radiation, we would be hardly pressed to determine the correlations between different modes as well. Therefore, in practice, quantum fluctuations act as a bath or environment for the macroscopic degrees of freedom of the Universe, and these evolve following a dissipative effective dynamics, whereby time reversal invariance is broken [10]. Under these circumstances, while we know that the present state of the Universe cannot be rendered totally independent of initial conditions, it makes sense again to ask for the relative likelihood of inflating cosmologies.

A similar set of questions have already been investigated in the framework of homogeneous cosmological models. In ref. [11] it has been shown that the back reaction of conformal particles created by a Taub Universe helps to delay recollapse [12], and thereby increases the likelihood of Inflation ( in these models, Inflation occurs when the Universe reaches a size such that the cosmological constant overpowers shear in the Hamiltonian constraint ). This was done by following the evolution self consistently, in the approximation were the back reaction of created particles is taken into account, but vacuum polarization terms are neglected.

In this paper, we shall study the relevance of semiclassical effects to Inflation in inhomogeneous but isotropic, with respect to a singled out point, models. In this context, to solve for the evolution self consistently is no longer possible. Rather, we shall follow the strategy of concentrating on a particular Cauchy surface, which is assumed to lie at the future of the semiclassical era, but at the past of the inflationary one. Thus, the Cauchy data on this surface are themselves the outcome of the semiclassical era. We shall study self consistent sets of Cauchy data, that is, solutions of Einstein's constraint equations, and obtain conditions for a set of data to lead to "natural" Inflation. We shall consider Inflation 
to be natural if there is a significative probability that, well after Inflation has begun, any nondescript observer will find him or herself into an Inflating, near Friedmann - Robertson - Walker like region. Then we shall show that these conditions are already contained in the requirement of consistence with an earlier semiclassical era. In other words, we shall conclude that semiclassical effects select Cauchy data leading to natural Inflation.

Our analysis shall proceed in the Hamiltonian, or ADM 13], formulation of General Relativity. Any spherically symmetric metric comprises two physical degrees of freedom, and two Lagrange multipliers, the lapse function, and the shift in the radial direction; the Klein - Gordon field introduces an extra degree of freedom. The Lagrange multipliers are associated to two nontrivial constraints, which in turn allow us to impose two arbitrary gauge conditions on the model ( we shall use this freedom to simplify the equations below, rather than opting for a gauge invariant formulation ). Our tactic shall be to use the constraints and the gauge freedom to fix entirely the space metric and the geometrodynamical momenta; the lapse and shift are then defined by the requirement of consistency of the dynamical Einstein's equations. This approach leaves the Klein - Gordon equation ( written in Hamiltonian form ) as the only dynamical law.

In this paper we shall keep a specific physical situation in mind. We found it convenient to take advantage of the gauge freedom in General Relativity to obtain this particular scenario in its simplest form. For this reason, we shall adopt a "custom made" gauge, rather than any of the usual choices, such as maximal slicing [13], a Tolman - like metric [14], or a synchronous gauge. For the same reason, we shall develop an analysis of the Einstein - Klein - Gordon system from first principles, rather than apply the general solutions available in the literature [15].

The situation of interest is as follows. As we have seen in our "back of the envelope" argument above, successfull chaotic Inflation requires a very high and homogeneous initial value of the field ( we shall not discuss here the possible sources of such field values; they occur in quantum cosmological models based on the Hartle - Hawking " no boundary " proposal [16]). Whatever the mechanism to provide such configuration, it is physically 
likely that quantum and/or thermal fluctuations will result in creating "holes" in the field, that is, regions where the value of the field gets closer to its ( vanishing ) equilibrium value. Of course a deep enough "hole" will not inflate at all, but a shallow "hole" may be capable of inflating, thus becoming an Inflationary island or bubble in a larger Universe. This island is, nevertheless, surrounded by a transition layer where conditions are far from homogeneity. The naturalness condition, over and above the usual conditions on the field for there to be Inflation, concerns the relative sizes of the island and the transition layer.

For simplicity, we shall consider a single island, so we shall assume that the field is high and homogeneous far from the origin. Under these conditions, the metric will approach asymptotically a Friedmann - Robertson - Walker ( FRW ) form. We shall use our gauge freedom to force the three metric to keep a FRW form everywhere. Departures from homogeneity shall be coded into the lapse and shift functions. As we shall see below, in this gauge we shall be able to display the dependence of these functions on the field and its conjugated momentum in detail. This, in turn, will allow us to translate the naturalness condition into a concrete inequality for the Cauchy data. In a number of cases of interest, such as when the field momentum vanishes, the field itself being arbitrary, we shall obtain closed form, exact expressions for lapse and shift. This is already a vast generalization over previously known results [6,7,9]; the general case can be handled perturbatively.

Allthough we shall not discuss the evolution of these Cauchy data in detail, we shall show below that the naturalness condition puts limits on the shift function accross the transition layer. On the other hand, as we shall discuss in more detail in the body of the paper, we expect the Universe to be conformally flat ( vanishing Weyl tensor ) by the end of the quantum era [17]. The exact form of the self consistent Cauchy data previously obtained shall allow us to show that, in this model, conformal flatness results in the same type of conditions than naturalness. In this sense, therefore, it can be said that semiclassical effects select for naturally inflating Cauchy data.

This result is both of relevance to cosmology, and of great interest as a non trivial application of quantum field theory in curved spaces (QFTCS). This theory being only an 
approximation to a yet unknown fully quantized theory of gravity, its meaningful applications are confined to weakly quantum effects, where gravitational quantum behavior is not expected to be relevant. Given these restrictions, QFTCS is only capable to yield entirely new results in phenomena where quantum effects are able to acummulate over time, there being no classical phenomena in a position to screen them. The canonical example where these conditions are fullfilled is Black Hole evaporation [18], which is still now possibly the main area of development in QFTCS. Conformal particle creation, from the vacuum, in cosmological settings, is similarly a cumulative, intrinsically quantum phenomenon. The study of the effect of particle creation processes on the shape of our Universe is therefore, beyond its relevance to cosmology, one of the few areas where QFTCS leads to observable predictions, not to be obtained in classical theory.

The paper is organized as follows. In next section we present the model and the solution of Einstein's constraint equations. In section III, we discuss the conditions for natural Inflation, the effect on Cauchy data of a semiclassical era, and the relationship between the two. In section IV we briefly state our conclusions. A technical discussion of the solution of the constraint equations for nonvanishing field momenta is included as an appendix.

\section{SELF CONSISTENT SPHERICALLY SYMMETRIC CAUCHY DATA}

\section{A. Canonical Variables and Hamiltonian}

In this section, we shall carry out an analysis of spherically symmetric solutions of the constraint equations of the Einstein - Klein - Gordon system, in order to discuss in the next section which Cauchy data eventually lead to acceptable inflationary cosmologies. We shall adopt for our discussion the ADM formalism, whose starting point is the $3+1$ decomposition of the space - time metric as

$$
d s^{2}=-N^{2} d t^{2}+g_{i j}\left(d x^{i}+N^{i} d t\right)\left(d x^{j}+N^{j} d t\right)
$$


Here, $g_{i j}$ is the metric induced on a space like surface, on which the $3+1$ decomposition is based, and $N, N^{i}$ are the lapse and shift functions which describe the embedding of the spatial surface into the four dimensional space time ( we follow MTW [13] conventions; latin indexes run from 1 to 3 ). Assuming spherical symmetry, we may simplify Eq.(四) to

$$
d s^{2}=-N^{2}(R, t) d t^{2}+A^{2}(R, t)(d R+\nu(R, t) d t)^{2}+B^{2}(R, t) d \Omega
$$

where we introduced $\nu=N^{1}$ and $d \Omega=d \theta^{2}+\sin ^{2} \theta d \varphi^{2}$. It is convenient to choose the conformal degree of freedom of the space metric as one of the geometrodynamical variables. Therefore, we rewrite Eq. (21) as

$$
d s^{2}=-N^{2}(R, t) d t^{2}+e^{2 \alpha(R, t)}\left\{X^{-4 / 3}(R, t)(d R+\nu(R, t) d t)^{2}+X^{2 / 3}(R, t) d \Omega\right\}
$$

The peculiar parametrization of the conformal metric will be of use below.

If we take $\alpha$ and $X$ as geometrodynamical canonical coordinates, then the canonical momenta shall be parametrized in terms of two independent degrees of freedom, $\Pi_{\alpha}$ and $\Pi_{X}$, canonically conjugated to the respective variables. The full momentum tensor density, in $R, \theta, \varphi$ coordinates, becomes

$$
\Pi_{j}^{i}=\left(\frac{1}{6}\right)\left[\Pi_{\alpha} \delta_{j}^{i}+\frac{3}{2} X \Pi_{X}\left(\delta_{j}^{i}-3 \delta_{1}^{i} \delta_{j}^{1}\right)\right]
$$

With this parametrization, the "kinetic" term in the Hamiltonian

$$
K=\left(\frac{16 \pi}{m_{p}^{2}}\right) N g^{-1 / 2}\left\{\Pi_{j}^{i} \Pi_{i}^{j}-(1 / 2)\left(\Pi_{i}^{i}\right)^{2}\right\}
$$

$\left(m_{p}\right.$ is Planck's mass $)$ becomes

$$
K=\left(\frac{16 \pi}{m_{p}^{2}}\right) \frac{N e^{-3 \alpha}}{24}\left\{-\Pi_{\alpha}^{2}+9 X^{2} \Pi_{X}^{2}\right\}
$$

The "shift" part of the Hamiltonian

$$
S=\Pi^{i j}\left(N_{i ; j}+N_{j ; i}\right)
$$

becomes 


$$
S=(1 / 3) \Pi_{\alpha}\left(\nu^{\prime}+3 \alpha^{\prime} \nu\right)-X \Pi_{X} \nu^{\prime}+X^{\prime} \Pi_{X} \nu
$$

where a prime means a derivative with respect to $R$. Finally, the "potential" term

$$
V=\left(-\frac{m_{p}^{2}}{16 \pi}\right) N g^{1 / 2} \mathcal{R}
$$

( where $\mathcal{R}$ is the spatial curvature ) is best computed by observing that the spatial metric is conformally flat. Indeed, introducing a new radial coordinate $r$ through

$$
\frac{d R}{X}=\frac{d r}{r}
$$

The spatial metric becomes $e^{2 \omega}\left[d r^{2}+r^{2} d \Omega\right]$, where

$$
\omega=\alpha+(1 / 3) \ln X-\ln r
$$

Therefore, we find

$$
\mathcal{R}=(-1) e^{-2 \omega}\left\{4 r^{-2} \partial_{r} r^{2} \partial_{r} \omega+2\left(\partial_{r} \omega\right)^{2}\right\}
$$

or, in terms of $R$ derivatives

$$
V=\left(\frac{m_{p}^{2}}{16 \pi}\right) N e^{\alpha} X^{4 / 3}\left[4 \alpha^{\prime \prime}+2 \alpha^{\prime 2}+\left(\frac{16}{3}\right) \alpha^{\prime}\left(\frac{X^{\prime}}{X}\right)+\left(\frac{4}{3}\right)\left(\frac{X^{\prime}}{X}\right)^{\prime}+\left(\frac{14}{9}\right)\left(\frac{X^{\prime}}{X}\right)^{2}-2 X^{-2}\right]
$$

The matter field introduces a new canonical variable $\phi$ and its conjugated momentum $\Pi_{\phi}$, and a new term in the Hamiltonian

$$
H_{m}=\nu \phi^{\prime} \Pi_{\phi}+\frac{N}{2}\left\{e^{-3 \alpha} \Pi_{\phi}^{2}+e^{\alpha} X^{4 / 3} \phi^{\prime 2}+e^{3 \alpha} m^{2} \phi^{2}\right\}
$$

where $m$ is the mass of the minimally coupled, real, non interacting field $\phi$. The total Hamiltonian is given by $K+S+V+H_{m}$.

\section{B. Field Equations and Gauge Conditions}

Having found the Hamiltonian of the model in the previous section, the field equations, in Hamiltonian form, are found by taking variational derivatives in the usual way. Variation with respect to lapse and shift yields the Hamiltonian and Momentum constraints 


$$
\begin{aligned}
& \left(\frac{16 \pi}{m_{p}^{2}}\right) \frac{e^{-3 \alpha}}{24}\left\{-\Pi_{\alpha}^{2}+9 X^{2} \Pi_{X}^{2}\right\} \\
& +\left(\frac{m_{p}^{2}}{16 \pi}\right) e^{\alpha} X^{4 / 3}\left\{4 \alpha^{\prime \prime}+2 \alpha^{\prime 2}+\left(\frac{16}{3}\right) \alpha^{\prime}\left(\frac{X^{\prime}}{X}\right)+\left(\frac{4}{3}\right)\left(\frac{X^{\prime}}{X}\right)^{\prime}+\left(\frac{14}{9}\right)\left(\frac{X^{\prime}}{X}\right)^{2}-2 X^{-2}\right\} \\
& +\frac{1}{2}\left\{e^{-3 \alpha} \Pi_{\phi}^{2}+e^{\alpha} X^{4 / 3} \phi^{\prime 2}+e^{3 \alpha} m^{2} \phi^{2}\right\}=0 \\
& (-1 / 3) \Pi_{\alpha}^{\prime}+\alpha^{\prime} \Pi_{\alpha}+\left(X \Pi_{X}\right)^{\prime}+X^{\prime} \Pi_{X}+\phi^{\prime} \Pi_{\phi}=0
\end{aligned}
$$

Variation with respect to the momenta yields the velocities

$$
\begin{gathered}
\dot{\alpha}=\frac{1}{3}\left(\nu^{\prime}+3 \nu \alpha^{\prime}\right)-\left(\frac{16 \pi}{m_{p}^{2}}\right)\left(\frac{N e^{-3 \alpha}}{12}\right) \Pi_{\alpha} \\
\dot{X}=-X \nu^{\prime}+X^{\prime} \nu+\left(\frac{16 \pi}{m_{p}^{2}}\right)\left(\frac{3 N e^{-3 \alpha}}{4}\right) X^{2} \Pi_{X} \\
\dot{\phi}=N e^{-3 \alpha} \Pi_{\phi}+\nu \phi^{\prime}
\end{gathered}
$$

( where a dot stands for time derivative ). Variation with respect to $\phi$ yields

$$
\dot{\Pi}_{\phi}=\left(\nu \Pi_{\phi}\right)^{\prime}+\left(N e^{\alpha} X^{4 / 3} \phi^{\prime}\right)^{\prime}-N e^{3 \alpha} m^{2} \phi
$$

which, toghether with Eq. (19), is equivalent to the Klein - Gordon equation. It is unnecessary to take variations with respect to $\alpha$ and $X$, as the resulting equations are dependent on those already derived.

We are thus left with six equations for eight unknowns, which must be supplemented with two arbitrary gauge conditions. As discussed in the Introduction, we envisage solutions which approach Friedmann - Robertson - Walker ( FRW) behavior at infinity, where the field shall be assumed to be homogeneous. This boundary condition shall be easiest to implement in a gauge where the three metric is constrained to be already in FRW form everywhere, so that deviations from homogeneity are encoded solely in the lapse and shift functions. Therefore we impose as gauge conditions

$$
\alpha^{\prime}=0
$$




$$
X=3 R
$$

The extreme simplicity of the functional dependence of $X$ is the reason behind our unconventional parametrization of the space metric ( the metric becomes explicitly FRW under the change of coordinates $\left.R=r^{3} / 3\right)$. These gauge conditions still allow for time redefinitions; the gauge can be totally fixed by demanding, e. g. , that the lapse function approaches 1 as $R \rightarrow \infty$.

The field equations acquire a simpler form in terms of the non canonical variables $P_{\alpha}=$ $e^{-3 \alpha} \Pi_{\alpha}, P_{X}=e^{-3 \alpha} \Pi_{X}$, and $P_{\phi}=e^{-3 \alpha} \Pi_{\phi}$. The constraints become

$$
\begin{aligned}
& \left(\frac{2 \pi}{3 m_{p}^{2}}\right)\left\{-P_{\alpha}^{2}+9(3 R)^{2} P_{X}^{2}\right\} \\
& +\frac{1}{2}\left\{P_{\phi}^{2}+m^{2} \phi^{2}+e^{-2 \alpha}(3 R)^{4 / 3} \phi^{2}\right\}=0 \\
& (-1 / 3) P_{\alpha}^{\prime}+3 R P_{X}^{\prime}+6 P_{X}+\phi^{\prime} P_{\phi}=0
\end{aligned}
$$

The dynamical equations for $\alpha$ and $X$ now become consistency conditions for the lapse and shift, namely

$$
\begin{gathered}
\dot{\alpha}=\frac{1}{3} \nu^{\prime}-\left(\frac{4 \pi}{3 m_{p}^{2}}\right) N P_{\alpha} \\
\nu^{\prime}-\frac{\nu}{R}=\left(\frac{36 \pi}{m_{p}^{2}}\right) R N P_{X}
\end{gathered}
$$

The Klein - Gordon equation takes the form

$$
\begin{gathered}
\dot{\phi}-\nu \phi^{\prime}=N P_{\phi} \\
\left(e^{3 \dot{\alpha}} P_{\phi}\right)=e^{3 \alpha}\left(\nu P_{\phi}\right)^{\prime}+e^{\alpha}\left(N(3 R)^{4 / 3} \phi^{\prime}\right)^{\prime}-N e^{3 \alpha} m^{2} \phi
\end{gathered}
$$

\section{Solving the constraint equations}

We turn now to the study of the solutions of the constraint and consistency equations derived in the previous subsection. To this end, it is convenient to introduce the notation 


$$
H^{2}=\left(\frac{4 \pi}{3 m_{p}^{2}}\right)\left[P_{\phi}^{2}+m^{2} \phi^{2}+e^{-2 \alpha}(3 R)^{4 / 3} \phi^{\prime 2}\right]
$$

In the homogeneous case, $H$ reduces to the Hubble constant. We also parametrize $P_{\alpha}$ and $P_{X}$ in terms of a new variable $\xi$, as follows

$$
\begin{gathered}
P_{\alpha}=-\left(\frac{3 m_{p}^{2}}{4 \pi}\right) H \cosh \xi \\
P_{X}=-\left(\frac{3 m_{p}^{2}}{4 \pi}\right) \frac{H}{9 R} \sinh \xi,
\end{gathered}
$$

whereby the Hamiltonian constraint is reduced to an identity, and the momentum constraint becomes

$$
\xi^{\prime}+\left(\frac{1}{2 R}\right)\left(e^{2 \xi}-1\right)=\frac{H^{\prime}}{H}+\left(\frac{4 \pi}{m_{p}^{2}}\right) \frac{\phi^{\prime} P_{\phi} e^{\xi}}{H}
$$

If the last term in Eq. (32) could be neglected, the general solution would be

$$
e^{2 \xi} \sim \frac{H^{2}}{\mathcal{H}^{2}+\frac{f}{R}}
$$

where $f$ is a constant, and

$$
\mathcal{H}^{2}=\frac{1}{R} \int_{0}^{R} d R^{\prime} H^{2}\left(R^{\prime}\right)
$$

$\left(\mathcal{H}^{2}\right.$ is therefore an smoothed out version of $\left.H^{2}\right)$. Indeed, we find

$$
\begin{gathered}
\mathcal{H}^{2 \prime}=\frac{H^{2}-\mathcal{H}^{2}}{R} \\
\xi^{\prime} \sim \frac{H^{\prime}}{H}-\left(\frac{1}{2 R}\right)\left[\frac{H^{2}-\left(\mathcal{H}^{2}+\frac{f}{R}\right)}{\mathcal{H}^{2}+\frac{f}{R}}\right],
\end{gathered}
$$

from where it is easy to verify Eq. (32). In the general case, we uphold the ansatz Eq. (33), but now allowing $f$ to be a function of $R$ and $t$; substituting into Eq. (32), we find

$$
f^{\prime}=-\left(\frac{8 \pi}{m_{p}^{2}}\right) R \phi^{\prime} P_{\phi} \sqrt{\mathcal{H}^{2}+\frac{f}{R}}
$$

To avoid a singularity at the origin, we must adopt the boundary condition $f(0)=0$. Therefore, in the case in which $P_{\phi}$ vanishes on the initial surface ( but the field profile is arbitrary ), or if the field is homogeneous, no matter the form of $P_{\phi}, f$ is identically zero, and our solution of the constraints is exact.

We shall return to the perturbative solutions to Eq. (37) in the Appendix. 


\section{Solving for Lapse and Shift}

Having reduced the constraints to the single Eq. (37) for the unknown function $f$, we now turn to consider the consistency conditions Eqs. (25), (26). We first notice they imply the simple relationship between lapse and shift

$$
\nu=3 R\left\{\dot{\alpha}-N H e^{-\xi}\right\}
$$

Replacing Eq. (38) into Eq. (26), we obtain

$$
R \frac{\left(N H e^{-\xi}\right)^{\prime}}{\left(N H e^{-\xi}\right)}=\frac{1}{2}\left(e^{2 \xi}-1\right)
$$

Now, Eqs. (33) and (35) can be combined to yield

$$
e^{2 \xi}-1=R\left\{\left(\ln \left[\mathcal{H}^{2}+\frac{f}{R}\right]\right)^{\prime}-\frac{f^{\prime}}{R\left(\mathcal{H}^{2}+\frac{f}{R}\right)}\right\}
$$

And so, using again Eqs. (33) and (37), and imposing the boundary condition that the lapse should approach 1 as $R \rightarrow \infty$, we find

$$
\begin{gathered}
N=e^{\left\{-\left(\frac{4 \pi}{m_{p}^{2}}\right) \int_{R}^{\infty} d R^{\prime}\left[\frac{\phi^{\prime} P_{\phi}}{\sqrt{\mathcal{H}^{2}+\left(\frac{f}{R}\right)}}\right]\left(R^{\prime}\right)\right\}} \\
\nu=3 R \dot{\alpha}\left\{1-\left(\frac{\sqrt{\mathcal{H}^{2}+\frac{f}{R}}}{\dot{\alpha}}\right) e^{\left(-\left(\frac{4 \pi}{m_{p}^{2}}\right) \int_{R}^{\infty} d R^{\prime}\left[\frac{\phi^{\prime} P_{\phi}}{\sqrt{\mathcal{H}^{2}+\left(\frac{f}{R}\right)}}\right]\left(R^{\prime}\right)\right)}\right\}
\end{gathered}
$$

These expressions, toghether with the gauge chosen form of $\alpha$ and $X$, completely determine the space time metric, once the field configuration is given. Allthough they are not yet in closed form, since the function $f$ is known only implicitly, as the solution of Eq. (37), they are explicit enough to serve our purposes. In the next section, we shall apply them to study the conditions under which natural Inflation occurs in the Universe.

\section{NATURAL INFLATION AND SEMICLASSICAL EFFECTS}

\section{A. Conditions for Natural Inflation}

In the previous section, we analyzed the Hamiltonian structure of spherically symmetric Einstein - Klein - Gordon systems, in a manner adapted to the further discussion of Inflationary cosmology, but keeping nevertheless full generality. In this subsection, we shall 
introduce a number of new assumptions, which will allow us to specialize the general formalism to an specific physical situation, a nonlinear perturbation of an otherwise successful chaotic inflationary model. We shall derive from this analysis the conditions under which Inflation may still be obtained after the perturbation.

Concretely, we have in mind a situation where, in an homogeneous cosmological model, a bubble is created, either through quantum or thermal tunneling, where the field is lower than its spatial average value. Since most bubbles are created in an instantaneously stationary configuration [19], we shall assume that $P_{\phi}$ vanishes in a neighborhood of the bubble. In the exterior region, notwithstanding, slow roll over conditions hold, and $P_{\phi} \sim-(3 \dot{\alpha})^{-1} N m^{2} \phi$. The field is homogeneous both within and outside the bubble [19]; for concreteness, we shall assume $\phi^{\prime} \geq 0$ throughout.

Let us first show how the expected result that inflation shall proceed both in and out the bubble, but at different rates, is recovered form the analysis of the previous section. In the exterior region, using the slow roll over value for $P_{\phi}$ in Eq. (37), we obtain

$$
f^{\prime} \sim\left(\frac{8 \pi}{3 m_{p}^{2} \dot{\alpha}}\right) R N m^{2} \phi \phi^{\prime} \sqrt{\mathcal{H}^{2}+\frac{f}{R}},
$$

which, in view of the definition of $H^{2}$, Eq. (29), can be reduced to

$$
f^{\prime} \sim\left(\frac{R N H^{2 \prime}}{\dot{\alpha}}\right) \sqrt{\mathcal{H}^{2}+\frac{f}{R}}
$$

The solution of this equation is

$$
f \sim R\left(H^{2}-\mathcal{H}^{2}\right)
$$

Indeed, for this form of $f$, we obtain $\xi=0$ ( cfr. Eq. (33)), $P_{X}=0$ ( cfr. Eq. (31)), and $P_{\alpha}=-\left(\frac{3 m_{p}^{2}}{4 \pi}\right) H$. From the consistency conditions Eqs. (25) and (26), and the requirement that Inflation obtains at infinity, we get $N \sim \dot{\alpha} / H$ and $\nu \sim 0$. Eq. (44) reduces to $f^{\prime}=$ $R\left(H^{2}\right)^{\prime}$, which is easily seen to follow from Eqs. (45) and (35). In regions where $H^{2}$ is essentially a constant, moreover, the cosmological time $\tau$ is related to coordinate time $t$ through $d t / d \tau=1 / N=H / \dot{\alpha}$, which shows that $H$ is indeed the "local" Hubble constant, as measured by a comoving observer. 
Let us consider now a neighborhood of the bubble. Since $P_{\phi} \sim 0$ here, we have $f \sim 0($ because of Eq. (37) and the boundary condition $f(t, 0)=0$ ). Also, from Eq. (41), we see that $N \sim$ constant $\sim \dot{\alpha} / H_{\text {out }}$, where $H_{\text {out }}$ is the value of $H$ just outside the transition layer. Inside the bubble proper, the field is homogeneous, and therefore $\mathcal{H} \sim H \sim$ constant $\equiv H_{\text {in }}$. Eq. (42) now gives

$$
\nu=3 R \dot{\alpha}\left\{1-\frac{H_{\text {in }}}{H_{\text {out }}}\right\}
$$

The full metric reads

$$
d s^{2}=-\left(\frac{\dot{\alpha}}{H_{\text {out }}}\right)^{2} d t^{2}+e^{2 \alpha}\left\{(3 R)^{-4 / 3}\left(d R+3 R \dot{\alpha}\left\{1-\frac{H_{\text {in }}}{H_{\text {out }}}\right\} d t\right)^{2}+(3 R)^{2 / 3} d \Omega\right\}
$$

Let us define a new radial variable

$$
\zeta=R e^{3 \int \dot{\alpha}\left\{1-\frac{H_{\text {in }}}{H_{\text {out }}}\right\} d t^{\prime}}
$$

In terms of $\zeta$, the metric becomes

$$
d s^{2}=-\left(\frac{\dot{\alpha}}{H_{\text {out }}}\right)^{2} d t^{2}+e^{2 \int \dot{\alpha}\left\{\frac{H_{\text {in }}}{H_{\text {out }}}\right\} d t^{\prime}}\left\{(3 \zeta)^{-4 / 3}(d \zeta)^{2}+(3 \zeta)^{2 / 3} d \Omega\right\}
$$

Which takes explicit FRW form under the further changes $d \tau=\left(\frac{\dot{\alpha}}{H_{\text {out }}}\right) d t, \zeta=(1 / 3) \rho^{3}$. We see that, as expected, Inflation is obtained inside the bubble, with $H_{\text {in }}$ as the Hubble constant measured by comoving observers.

We conclude that, for successful Inflation, the usual conditions of high and homogeneous field must hold inside the bubble. Moreover, if the bubble is several horizons in size originally, it cannot recollapse entirely, since the wall of the bubble cannot exceed the speed of light, but the Hubble flow is not so limited. An observer deep inside the bubble, therefore, will be allowed to assume that he or she is living in a FRW Universe, irrespective of the conditions outside the bubble.

Conditions in and outside the bubble are linked through the requirement of naturalness, however. In the simplified model we have considered, we obtained two inflationary regions, the interior and exterior of the bubble, separated by a transition layer. In a more realistic 
model, we would consider a Universe composed of many bubbles, each with its own surrounding wall. Naturalness is the requirement that, at any generic instant, the volume inside the bubbles should be a significative fraction of the volume inside the walls ( indeed, if we were to carry the Copernican principle to extremes, we should demand that the volume within the bubbles be much larger than the volume outside them ). A similar condition, easier to implement, is that each bubble should be comparable in size to the wall surrounding it.

Let us apply the later version of naturalness to our model. As an estimate of the size of the wall at any given moment, we shall use the forward light cone of the original transition layer. This overestimates the actual wall, but is nevertheless appropiate to our purposes, because observers within the light cone can see the wall, and therefore are aware that they do not live in a FRW cosmology.

To follow the evolution of the light cone it is not necessary to solve the Klein Gordon equation in any detail. On the contrary, by continuity, the limit light rays emerging from the walls shall behave essentially like the light rays of the Inflationary regions, and these are essentially slowly evolving de Sitter geometries. Concretely, the light cone shall expand a distance of overall $H_{\text {out }}^{-1}$, measured in the $r$ coordinate, into the exterior region, and of $H_{i n}^{-1}$, measured in the $\rho$ coordinate, into the bubble ( recall that $r$ and $\rho$ are the coordinates in which the metric takes explicitly the usual FRW form, outside and inside the bubble, respectively ).

To compare the relative sizes of bubble and wall, however, we must use an uniform coordinate system. If we choose the $r$ coordinate, for example, we see that the line $\rho \sim \rho_{0} \equiv$ constant marking the inner boundary of the wall, becomes

$$
r=\rho_{0} e^{-\int \dot{\alpha}\left\{1-\frac{H_{\text {in }}}{H_{\text {out }}}\right\} d t^{\prime}}
$$

So, for most times, the bubble is actually exponentially small with respect to the wall ( since we are assuming that the expansion rate inside the bubble is lower than outside ). Of course, we do not require the bubble to be comparable to the wall for all times, but only for the 60 or so e - foldings that Inflation lasts. So, in this model, naturalness means that 
the exponential contraction of the bubble should not be too noticeable for the first 60 e foldings of Inflation. The necessary condition for this, as follows from Eq. (50), is

$$
\left\{1-\frac{H_{\text {in }}}{H_{\text {out }}}\right\} \leq \frac{1}{60}
$$

Only Cauchy data satisfying this condition shall lead to natural Inflation, even if they otherwise fulfill the requirements for enough Inflation in and out the bubble, separately. From the point of view of classical cosmology, however, naturalness, requiring a correlation between regions several horizons apart, can only be imposed through fine tuning of initial conditions. The possibility remains, however, that semiclassical effects actually favor initial conditions, for the classical era, already satisfying Eq. (51). In order to investigate this possibility, we turn now to a discussion of the relevance of semiclassical effects in cosmology.

\section{B. Semiclassical Effects and Natural Inflation}

As discussed in the Introduction, our goal is to determine whether the consideration of semiclassical cosmological effects turns naturally inflating Cauchy data any more likely than simply to assume them ad hoc. For this to be the case, it is necessary that semiclassical effects should break the one to one correspondence between the cosmic states before and after the semiclassical era ( or, more exactly, that semiclassical effects should invalidate Liouville's Theorem as applied to the Universe ). It follows from this that semiclassical effects resulting merely in the replacement of the Einstein - Hilbert action by a more complicated, but still real and local, effective action, will not affect the likelihood of natural Inflation and can be disregarded.

The situation changes, however, when we consider particle creation processes [20]. Indeed, under suitable statistical assumptions, particle creation defines an arrow of time [10,21]. More important to our present concern, the back reaction of the created particles has a smoothing effect on the evolution, thus making certain field configurations ( those

leading to no creation ) prefered above others [20,22]. Thus, to quote a well known example, 
the possibility of conformal particle creation makes an isotropic universe a prefered alternative to anisotropic ones. For simple cosmological models, such as Bianchi type I universes [17], it is actually possible to follow in detail the process of particle creation and isotropization. Moreover, it has been shown that the connection between these phenomena is not limited to the semiclassical era, extending as well to the fully quantum one [10,23].

The details of how particle creation proceeds in a given model depend on the peculiar matter content of the model. In the absense of a generally accepted theory of elementary particle physics, there is no absolute criteria for what a realistic theory should look like. However, as far as we are mostly concerned with processes occurring early on in the semiclassical era, we can make some simplifications. Indeed, the main effects of a strong gravitational field on elementary particles can be described by allowing masses and coupling constants to run according to their renormalization group equations, the scale being fixed by suitable curvature invariants [24,25]. It follows that asymptotically free theories of elementary particles actually become free in the early Universe, and that masses can be ignored. For spin $1 / 2$ and 1, minimally coupled fields, this implies they become approximately conformally invariant.

For scalar fields the situation is slightly more complex, because, while conformal coupling is a fixed point of the 1 loop Renormalization Group equations, higher loop corrections tend to make it unstable [24]. These small deviations from conformal invariance are not important, nevertheless, because, in any case, creation of scalar particles is less efficient than that of gauge and spinor fields.

The creation rate for conformal particles in nearly conformally flat Universes is given by $N C^{2} / 80 \pi$, where $C^{2}$ is the square of the Weyl tensor, and $N$ the effective number of particle species, defined as $N=N_{1}+N_{1 / 2} / 4+N_{0} / 24, N_{i}$ being the number of species of spin $i$ [17]. The value of $N$ depends upon the particular theory of elementary particles being used; for typical GUTs, $N \sim 100$ to 1000 [26].

In view of the earlier discussion, any initial configuration with a nonzero Weyl tensor will be brought towards conformal flatness by particle creation; correspondingly, towards 
the end of the quantum era, the Weyl tensor should be negligible against the conformal part of the curvature. In a near inflationary evolution, the curvature scale is set by the Hubble constant, and our argument shows that the inequality $C^{2} H^{-4} \ll 1$ must hold.

To compare this constraint on physically acceptable Cauchy data to the condition for natural Inflation, Eq. (51) above, we must compute the Weyl tensor for the specific form of the metric corresponding to a neighborhood of the bubble. Assuming slow roll over conditions, that is, that space derivatives dominate over time derivatives, except for the conformal factor, we obtain

$$
C^{2}=12 H_{\text {out }}^{4}\left\{R \beta^{\prime}-3 R^{2 / 3}\left[R^{4 / 3} \beta \beta^{\prime}\right]^{\prime}\right\}^{2} \sim 12 H_{\text {out }}^{4}\left\{R \beta^{\prime}\right\}^{2}
$$

where

$$
\beta=\left\{1-\frac{\mathcal{H}}{H_{\text {out }}}\right\}
$$

From Eq. (35) above, it is easy to see that

$$
R \beta^{\prime}=-\frac{H^{2}-\mathcal{H}^{2}}{2 H_{\text {out }} \mathcal{H}}
$$

Recall now that $H_{\text {out }}$ is the limit value of $H$ as we approach the edge of the transition layer. There is then a region, close to the outer rim of the layer, where $H \sim H_{\text {out }}$. In this region

$$
R \beta^{\prime}=-\beta\left[\frac{1-\beta / 2}{1-\beta}\right] \sim-\beta
$$

and the condition of smallness on the Weyl tensor translates into $\beta \ll 1$. But recall also that, from its definition, $\mathcal{H}$ is slowly varying compared to $H$ itself. Therefore, across the transition layer, even if already $H \sim H_{\text {out }}$, we still have $\mathcal{H} \sim H_{\text {in }}$, and $\beta \ll 1$ actually implies the condition Eq. (51) for natural inflation.

To summarize, we have shown that, even if a mechanism were found to create regions of large, homogeneous inflaton field, classical Inflation would still be unnatural, because the volume of the transition layers among these regions would be typically larger than the regions themselves. However, semiclassical effects forbid Cauchy data where the local Hubble 
constant changes strongly between inflating regions. Therefore, in semiclassical cosmology, successful Inflation is also natural, without the need of extra assumptions correlating the value of the Inflaton field in the different inflating domains. In this sense, semiclassical Inflation is more natural than its classical counterpart.

\section{CONCLUSIONS}

The discussion in this paper has progressed in three stages, each using the results of the previous one, but still essentially independent. In the first stage, we analyzed the constraint equations of General Relativity coupled to a Klein - Gordon field. Assuming spherical symmetry, we showed that the general solution to these constraints could be expressed in terms of a single unknown function. In many cases of interest, this function may be determined exactly, yielding consistent Cauchy data in closed, analytic, form. In the general case, the unknown function may be computed perturbatively.

This result has been achieved through the use of a special gauge choice, devised to simplify the constraint equations to the utmost. The only dynamical law in this approach is the Klein - Gordon equation itself. The gain in simplicity of the constraints is paid for in terms of the complexity which this equation acquires. However, being hyperbolic, the Klein - Gordon equation is amenable to a qualitative treatment, which discloses the general features of the cosmological evolution.

In the second stage of our reasoning, we developed such a qualitative analysis, seeking to determine the necessary conditions for what we called "natural" inflation. Of course, to obtain Inflation at all certain special initial conditions must be assumed, involving very high and homogeneous initial values of the inflaton field. Our discussion aimed to show that, even if these conditions were assumed locally, the resulting Universe would still be very different to an Inflationary one, unless one further condition were added, "naturalness", linking the values of the field over several horizon lenghts. This result is not dependent upon the full

details of the Cauchy data previously found; however, knowledge of those data allowed us 
to translate naturalness into a concrete inequality, which the Cauchy data must satisfy, in order to lead to an admissible cosmology.

Finally, in the third stage of our argument we confronted our conditions for "natural" Inflation against the present understanding of semiclassical cosmology. Based on the well proven smoothing effect of particle creation, we argued that the Universe should have left the semiclassical era in a state of near conformal flatness. This already puts a bound on the possible gradients of the metric elements at the beginning of Inflation; our explicit form for those metric elements allowed us to show that this bound actually implies the naturalness condition previously derived.

There is an important caveat which goes with this argument, and which we would like to make explicit here. In discussing the likely evolution of the Universe during the semiclassical era, we are already assuming that the initial conditions at its beginning were not too extreme. Indeed, the detailed models of semiclassical cosmologies in the literature assume for the most part near Friedmann - Robertson - Walker conditions [27], and it would be unjustified to extrapolate these results to arbitrarily strong inhomogeneities. Moreover, it should not be expected that semiclassical effects could allways stabilize a classically growing perturbative mode.

However, even under the most conservative reading of the literature, it should be accepted that, under the statistical assumptions discussed in the Introduction, semiclassical evolution is irreversible, and, in particular, that a nontrivial set of initial conditions is actually brought to conformal flatness through particle creation and back reaction. These results are enough to support our main conclusion, which is that semiclassical effects enhance the likelihood of Inflation in the Early Universe. This conclusion, in turn, confirms the findings of previous studies of inflation in homogeneous models [11].

It is certainly likely that a conclusion along these lines, given these hypothesis, could have been reached through general arguments, independent of the detailed form of the Cauchy data. However, the particular strategy we have followed is relevant in that it points the way for further studies of classical and semiclassical Inflation. For example, the self 
consistent Cauchy data for the Einstein - Klein - Gordon system we present here, provide also an environment where such questions as the dependence on initial conditions of the spectrum of density fluctuations in Inflation can be investigated. The relevant feature of the solutions presented here is, of course, that in no way we have assumed small departures from homogeneity.

We are continuing our research on these manyfold questions.

\section{ACKNOWLEDGEMENTS}

We would like to acknowledge the multiple comments and suggestions from the participants to the NATO Workshop on Origin of Structure in the Universe ( Pont d'Oye, Belgium, 1992), specially L. Grishchuk, A. Starobinsky, W. Unruh and R. Wald, where a preliminary draft of this work was presented.

E. C. wishes to thank the hospitality of the RGGR group at the Free University ( Brussels ); M. S. that of the GTCRG group at IAFE ( Buenos Aires ).

This work was supported by CONICET, UBA and Fundación Antorchas ( Argentina ), and by the Directorate General for Science Research and Development of the Comission of the European Communities under contract No CI1-0540-M(TT).

\section{APPENDIX: PERTURBATIVE SOLUTIONS TO THE CONSTRAINTS}

In the main body of the paper we have shown that the full geometry can be parametrized in terms of a single function $f$, obeying Eq. (37). In particular cases, such as when $P_{\phi}$ vanishes identically, $f \equiv 0$, and the metric can be worked out explicitly. However, as we pointed out above, it would be unrealistic to assume such conditions throughout space. For successfull inflationary models, moreover, we must have $f \sim R\left(H^{2}-\mathcal{H}^{2}\right)$ for large $R$. It is interesting, therefore, to investigate the solutions to Eq. (37) in non trivial cases.

If we assume, as in the main body of the paper, $\phi^{\prime} \geq 0$ and $P_{\phi} \leq 0$ ( in Inflation, $\phi^{\prime}=0$ and $\left.P_{\phi} \sim-m^{2} \phi / 3 H\right)$, then $f^{\prime}$ is positive, and $f$ is a non decreasing and non 
negative function of $R$. Under these conditions, it is possible to build a sequence of functions approximating $f$ as follows: the first function $f_{0}$ in the sequence is identically zero, and the n-th is the solution to

$$
f_{n}{ }^{\prime}=-\left(\frac{8 \pi}{m_{p}^{2}}\right) R \phi^{\prime} P_{\phi} \sqrt{\mathcal{H}^{2}+\frac{f_{n-1}}{R}}
$$

With boundary condition $f_{n}(0)=0$.

We observe that all functions in the sequence are positive and non decreasing. The sequence itself is nondecreasing at every point: $f_{1}$ is certainly larger than zero, because it starts at zero, and has a positive derivative; $f_{2}$ is larger than $f_{1}$, because both are positive, nondecreasing functions, with the same value at the origin, and $f_{2}$ has the larger derivative, etc. Therefore, the sequence $f_{n}(R)$ has a limit for every $R$, and the limit function satisfies Eq. (37).

This method of successive approximations is appealing not only because convergence is guaranteed, but also because at every step it is possible to estimate its accuracy. Indeed, from Eqs. (37) and (56) we see that

$$
\left(f-f_{n}\right)^{\prime}=-\left(\frac{8 \pi}{m_{p}^{2}}\right) R \phi^{\prime} P_{\phi}\left\{\sqrt{\mathcal{H}^{2}+\frac{f}{R}}-\sqrt{\mathcal{H}^{2}+\frac{f_{n-1}}{R}}\right\}
$$

Since the square root is a convex function, it follows that

$$
\left(f-f_{n}\right)^{\prime} \leq-\left(\frac{8 \pi}{m_{p}^{2}}\right) \phi^{\prime} P_{\phi}\left\{2 \sqrt{\mathcal{H}^{2}+\frac{f_{n-1}}{R}}\right\}^{-1}\left(f-f_{n-1}\right)
$$

And a fortiori

$$
\left(f-f_{n}\right)^{\prime} \leq h(R)\left(f-f_{n-1}\right)
$$

where

$$
h(R)=-\left(\frac{4 \pi}{m_{p}^{2}}\right) \frac{\phi^{\prime} P_{\phi}}{\mathcal{H}}
$$

Eq. (59) can be rewritten as

$$
\left(f-f_{n}\right)^{\prime}-h(R)\left(f-f_{n}\right) \leq h(R)\left(f_{n}-f_{n-1}\right)
$$


which in turn leads to

$$
f \leq f_{n}+\int_{0}^{R} d R^{\prime} h\left(R^{\prime}\right) e^{\int_{R^{\prime}}^{R} d R^{\prime \prime} h\left(R^{\prime \prime}\right)}\left(f_{n}-f_{n-1}\right)
$$

This is the sought for bound on $f$ 


\section{REFERENCES}

* E - mail address: Calzetta@iafe.edu.ar

† E - mail address: Pnardon@ulb.ac.be

$\ddagger$ Present Address: Physics Department, University of Tours, France.

${ }^{1}$ S. Weinberg, Gravitation and Cosmology (Wiley, New York, 1972).

${ }^{2}$ E. Kolb and M. Turner, The Early Universe ( Addison - Wesley, New York, 1990 ).

${ }^{3}$ G. Smoot et al. , Structure in the COBE DMR First Year Maps, Astrophys. J. Lett., to appear.

4 A. Guth, Phys. Rev D23, 347 (1981).

5 J. Bardeen, Phys. Rev. D22, 1882 (1980); J. Frieman and C. Will, Astrophys. J. 259, 437 (1982); H. Feldman and R. Brandenberger, Phys. Lett 227B, 359 (1989); 220B, 361 (1989); D. Salopek and D. Bond, Phys. Rev. D43, 1005 (1991).

${ }^{6}$ H. Kurki - Suonio, J. Centrella, R. Matzner and J. wilson, Phys. Rev. D35, 435 (1987).

7 T. Piran and R. Williams, Phys. Lett. 163B, 331 (1985); T. Piran, ibid. 181B, 238 (1986); D. Goldwirth and T. Piran, Phys. Rev. D40, 3263 (1989); D. Goldwirth, Phys. Lett. 243B, 41 (1990); D. Goldwirth and T. Piran, Phys. Rev. Lett. 64, 2852 (1990); D. Goldwirth, Phys. Rev. D43, 3204 (1991);

8 A. Linde, Phys. Lett. 129B, 177 (1983).

9 E. Calzetta and M. Sakellariadou, Phys. Rev D45, 2802 (1992).

10 B. L. Hu, Physica A158, 399 (1989); E. Calzetta and B. L. Hu, Phys. Rev. D40, 656 (1989).

11 E. Calzetta, Phys. Rev D44, 3043 (1991).

12 X. Lin and R. Wald, Phys. Rev. D40, 3280 (1989);D41, 2444 (1990). 
${ }^{13}$ C. Misner, K. Thorne and J. Wheeler, Gravitation ( Freeman, San Francisco, 1973).

${ }^{14}$ L. Landau and E. Lifschitz, Classical Theory of Fields (Pergamon, London, 1975).

15 B. Berger, D. Chitre. V. Montcrieff and Y. Nutku, Phys. Rev. D5, 2467 (1972); W. Unruh, Phys. Rev. D14, 870 (1974); see also the treatment of spherically symmetric Cauchy data in P. Bizon, E. Malec and N. Ó Murchadha, Class. Q. Grav. 6, 961 (1989); Phys. Rev. Lett. 61, 1147 (1988); U. Brauer and E. Malec, Phys. Rev. D45, R1836 (1992); E. Malec and N. Ó Murchadha, Trapped Surfaces and Spherically Closed Cosmologies, preprint (1992).

16 S. Hawking, in 300 Years of Gravitation, edited by S. Hawking and W. Israel ( Cambridge University Press, Cambridge (UK), 1987).

17 Ya. B. Zel'dovich and A. Starobinsky, JETP Lett. 26, 252 (1977); B. L. Hu and L. Parker, Phys. Rev. D17, 933 (1978); J. Hartle and B. L. Hu, ibid. D20, 1772 (1979); E. Calzetta and B. L. Hu, ibid. D35, 495 (1987); J. P. Paz, ibid. D41, 1054 (1990).

18 S. Hawking, Comm. Math. Phys. 43, 199 (1975).

19 S. Coleman, Phys. Rev. D15, 2929 (1977), and Aspects of Symmetry ( Cambridge University Press, Cambridge (UK), 1985).

${ }^{20}$ L. Parker, Phys. Rev. Lett. 21, 562 (1968); Phys. Rev. 183, 1057 (1969); in Asymptotic Structure of Space Time, edited by F. Esposito and L. Witten ( Plenum, New York, 1977 ) .

${ }^{21}$ B. L. Hu and H. Kandrup, Phys. Rev. D35, 1776 (1987); H. Kandrup, ibid. D37, 3505 (1988); ibid. D38, 1773 (1988).

${ }^{22}$ Ya. B. Zel'dovich, in Magic without Magic, edited by J. Klauder ( Freeman, San Francisco, 1972 ), p. 277; L. Parker, Phys. Rev. Lett. 50, 1009 (1983).

23 E. Calzetta, Phys. Rev. D43, 2498 (1991); E. Calzetta, M. Castagnino and R. Scocci- 
marro, ibid. D45, 2806 (1992).

${ }^{24}$ L. Parker and D. Toms, , Phys. Rev. Lett. 52, 1269 (1984); Phys. Rev. D29, 1584 (1984).

${ }^{25}$ E. Calzetta, I. Jack and L. Parker, Phys. Rev. Lett. 55, 1241 (1985); Phys. Rev. D33, 953 (1986), (E) D34, 1235 (1986); E. Calzetta, Ann. Phys. (NY) 166, 214 (1986).

${ }^{26}$ G. G. Ross, Grand Unified Theories ( Benjamin / Cummings, Menlo Park, 1985).

27 Among the few exceptions let us quote B. L. Hu, Phys. Rev. D8, 1048 (1973) and D9, 3263 (1974). 